\title{
PROCEEDINGS OF THE SEVENTEENTH ANNUAL MEETING OF THE AMERICAN SOCIETY FOR CLINICAL INVESTI- GATION HELD IN WASHINGTON, D. C., MAY 4, 1925
}

Cultivation of Vaccine Virus in Artificial Media. By RoBert N. Nye and (by invitation) FrederICK PARKer, JR., Boston, Mass.

Continuing the work of Parker it has been possible to cultivate vaccine virus in artificial media at $37.5^{\circ} \mathrm{C}$. for 130 days. The medium is essentially a living tissue culture, consisting of a small bit of normal rabbit testis to which is added infected testis (or plasma), the whole being held in place by one or two drops of diluted normal rabbit plasma. The cultures are made in small Petri dishes (5 $\mathrm{cm}$. diameter) and after coagulation of the plasma the dishes are inverted and sealed with sterile vaseline. Transplants are made every five or six days. Analysis of the air in the Petri dishes after five to six days incubation shows an oxygen content comparable to that of normal air. Intradermal titrations in rabbits show a marked increase in the actual amount of virus from generation to generation, thus eliminating the possibility of mere survival.

Preliminary Observations on the Toxin of Streptococcus erysipelatis. By H. L. Amoss and (by invitation) KonRad E. BirkHaug, Baltimore, Md.

The sterile Berkefeld filtered urine of rabbits with experimental crysipelas and of patients early in the acute stage of erysipelas gives a reaction when injected into the skin of some normal rabbits and especially into rabbits having an active experimental erysipelas lesion. The skin-reacting substance is heat labile and neutralizable by anti-erysipelas serum and quickly disappears from the urine during recovery.

The toxin was produced by anaerobic growth of organisms in tryptic broth. One cubic centimeter killed rabbits weighing 1500 grams. Weaker toxic filtrates were concentrated by precipitation with $\left(\mathrm{NH}_{4}\right)_{2} \mathrm{SO}_{4}$ at $\mathrm{pH}$ 4.6. The power of these streptococci to produce toxin does not parallel their hemolysin production. Anti-scarlatinal serum does not neutralize the toxin. Anti-erysipelas serum does not give Schultz-Charlton reaction in scarlet fever.

There is some evidence that erysipelas toxin is produced by a ferment elaborated by Streptococcus erysipelatis.

Twenty rabbits received weekly injections of toxic filtrate either intravenously, subcutaneously, intramuscularly, or intracutaneously for four months. The serum of these rabbits acquired no antitoxic properties and the rabbits themselves were just as susceptible to intravenous and intracutaneous injection of toxic filtrate and of living erysipelas streptococci as normal rabbits. It appears that 
the toxin in rabbits is not antitoxigenic and an analogy to the $S$ factor of pneumococcus is suggested.

Observations on the Amount of Scarlatinal Antitoxin Required to Cure Scarlet Fever. By James D. Trask (by invitation) and Francis G. Blake, New Haven, Conn. The goal to be attained in the treatment of scarlet fever with antitoxin is the neutralization of toxin and establishment of an excess of antitoxin in the blood of the patient. The amount of serum required will vary with the size of the patient, the severity of the disease, and the antitoxin content of the serum. To determine the amount of serum necessary the presence of toxin and antitoxin in the blood before and after intramuscular treatment has been determined in 42 cases of varying ages and severity, treated with 9 different lots of serum of varying antitoxin content.

The presence of toxin was determined by the capacity of the patient's serum to produce a local skin reaction in susceptible volunteers; the presence of antitoxin, by a positive blanching test. The antitoxin content of the serums was measured by determining the minimum amount of serum that would produce a positive blanching test.

The results show that a serum should contain at least 12,500 minimum blanching doses per cubic centimeter to be therapeutically efficient. This is equivalent to at least 10,000 skin test neutralizing doses per cc. or 100 units. Maximum doses are 3000 to 8000 units for children, 3000 to 12,000 for adults.

Experimental Pneumonia in Mice Following Inhalation of Streptococcus hemolyticus and of Friedlander Bacillus. By ERNEST G. STILLMAN and (by invitation) ARNold Branch, New York, N. Y.

The reaction of mice to inspired bacteria varies according to the kind of organism inspired.

Mortality and actual infection bear a direct relationship to the length of time the inspired bacteria persist in the lungs following inhalation. Hemolytic streptococci and B. Friedlander which have reached the lungs of normal mice following inhalation persist for several days. A fatal septicemia, with or without pulmonary localization, is frequent, and death may not occur for several days following exposure.

Pneumococci, on the other hand, rapidly disappear from the lungs of normal mice following inhalation and rarely cause a septicemia. In alcoholized mice, however, the pneumococci persist in the lungs for a longer period, a fatal septicemia is frequent, and pulmonary localization of the infection only occurs in partially immunized animals. Death occurs early, generally within 5 days.

On Certain Pharmacodynamic Actions of Bacterial Poisons. By KARL K. KoessLer and (by invitation) JulIAN H. LEwIS, Chicago, Ill.

In the last ten years the senior author and his collaborators concentrated their attention on the chemical study of the poisons which are formed when micro- 
organisms act upon proteins and amino acids. Quantitative chemical methods were devised which made it possible to separate these poisonous amines and to determine the amounts produced. It was shown that microorganisms which form histamine and tyramine are normal inhabitants of the human intestinal tract. Recent still unpublished investigations showed that this faculty of decarboxylation leading to amine production is not restricted to intestinal bacteria. The mixture of common pyogenic microorganisms contained in the bronchial expectoration, in tonsils and empyema fluids is able to form large quantities of these poisonous amines, which were pharmacologically characterized by their selective action on the smooth muscle fibre system. The question suggested itself naturally whether other poisons of similar constitution are not formed under our experimental condition?

This and other considerations made it desirable to study by delicate physiological methods bacterial culture filtrates and their various fractions obtained by chemical separation in the hope of elucidating, perhaps, the large problem of the factor of intoxication in bacterial diseases.

A method of determining bronchospasm in the guinea pig and a second method of demonstrating arterial constriction in vitro were devised. By means of these two methods the authors were able to show that the common microorganisms contained in the bronchial exudate, chiefly streptococci and pneumococci, produce chemically still unidentified poisons, which frequently have an intense constrictor action on the bronchioles and others which have definite vasoconstrictor action. The relationship of these experiments to human pathology $(a)$ to bronchospastic forms of dyspnoea of non-allergic nature and $(b)$ to the first stage of arterial hypertension, arterial spasm, is suggestive.

\section{Hay Fever. The Results of Treating the Same Patient in Four or More Successive}

Years. By Francis M. Rackemann, Boston, Mass.

A study of hay fever restricted to those patients with late hay fever due to ragweed, who were treated during several years, eliminates questions of pollen specificity and of widely different degrees of hypersensitiveness.

Skin tests done at the outset of each season show a remarkably constant degree of hypersensitiveness in 85 per cent of the 54 patients. The relation of the details of the treatment to the end result is best shown by curves which appear when the doses in each year are plotted against dates and amounts. The striking parallelism of these curves indicates again a degree of hypersensitiveness constant from year to year.

Similar courses of treatment lead to similar end results. Where however, the courses are different, it is possible to show that in some cases good results occur with a larger amount of treatment, while in other cases, excessive dosage, particuularly if accompanied by large local or general reactions, leads to results less favorable than those in other years when the dosage was smaller.

This indicates that success depends upon a course of doses the amounts of which are optimal for the individual patient. 
Contribution to the Chemotherapy of Protozoal Infections. By GeORGe BAEHR, New York, N. Y.

Observation of cases of amebic colitis resistant to most drastic treatment with emetin hydrochloride and emetin bismuth iodide necessitated search for another agent which would not only destroy organisms within the intestinal lumen but would possess sufficient penetrating power to kill off amebae imbedded within the mucous membrane. The extreme sensitiveness of amebae to ultraviolet radiation suggested the utilization of secondary $x$-rays produced by Roentgen radiation of the abdomen after previous filling of the colon with fluorescent material.

An adequate agent seems to have been found in a combination of flumerin, a mercury fluorescein preparation invented by White as a spirochaetocide, and roentgen rays. Prompt and seemingly permanent disappearance of amebae was secured in chronic cases of years duration with rapid healing of ulcers and permanent disappearance of symptoms.

Intravitam experiments in amebic colitis and in-vitro experiments with paramecia suggest that the generation of secondary $x$-rays within the intestine probably plays little if any rôle. The phenomenon appears analogous to that discovered over 25 years ago by $\mathrm{v}$. Tappeiner, who observed that the sensitiveness of protozoal organisms to fluorescent substances is enormously increased in the presence of sunlight. The possibility of a mercurial effect has not been excluded.

Effects of Arsenic Injections on Endothelial Permeability. By William F. PeterSEN and (by invitation) T. P. Hughes, Chrcago, Ill.

Therapeutic doses of arsenic (in normal dogs) produce a primary stimulation of endothelium. This is of short duration and seems largely confined to increased permeability for water. This is followed by a period of diminished permeability both for water and for larger protein aggregates (hemoglobin). We believe that this variation is partly responsible for the change of weight of patients under arsenic medication. With toxic doses the primary increase is not only for water but for colloids. Even in these cases there is a short period of recovery. Then follows a final period of increased permeability with other evidences of cellular injury (bile and erythrocytes in the lymph, as well as increase of sugar and phosphates). This final period seems to be irreversible and is the basis for the toxic manifestations of arsenic poisoning.

Studies of the Capillaries and Thebesian Vessels of Human and Cat Hearts. By Joseph T. WEarn, Boston, Mass.

Intracardiac injections of India ink or dyes in living cats under various conditions have made possible the quantitation of the capillaries of the heart. A normally beating heart so injected may show an even distribution of 2000 to 3500 capillaries per square millimeter, and following the injection of histamine the number may be as high as $\mathbf{5 7 0 0}$ per square millimeter. 
Cat heart after histamine

Capillaries per square millimeter

\begin{tabular}{c|c|c|c|c}
\hline Left ventricle & Right ventricle & Pap. muscle & Septum & Auricle \\
\hline 5,728 & 5,616 & 4,700 & 5,614 & 4,800 \\
\hline
\end{tabular}

These findings strongly suggest a reserve supply of capillaries in the heart.

Perfusion of the coronary arteries was carried out and the quantity of outflow from all sources follows:

\begin{tabular}{c|c|c|c|c}
\hline Experiment & \begin{tabular}{c} 
From Thebesian \\
vessels of right \\
auricle and ventricle \\
\cline { 1 - 2 }
\end{tabular} & $\begin{array}{c}\text { From left auricle } \\
\text { and ventricle }\end{array}$ & $\begin{array}{c}\text { Coronary sinus } \\
\text { and veins }\end{array}$ & $\begin{array}{c}\text { Leaks from cut } \\
\text { surfaces }\end{array}$ \\
\cline { 1 - 3 } 1 & $c c$. & $c c$. & $c c$. & cc. \\
2 & 250 & 235 & 50 & 80 \\
3 & 142 & 100 & 37 & 47 \\
4 & 235 & 175 & 20 & 120 \\
\hline
\end{tabular}

In perfusing the coronary sinus and veins, all the perfusate escaped through the Thebesian vessels, while the perfusate through the Thebesian vessels escaped through the coronary sinus.

None of these procedures gave a good injection of the capillaries but injected larger vessels completely. Perfusion of the Thebesian vessels injected vessels similar to those injected when perfusing the coronary arteries.

All these findings hold true for a dead cat's heart and show the existence of a large direct connection between the coronary arteries and the chambers of the heart, in addition to the well known communication between the Thebesian vessels and the veins.

The Effect of Caffein Sodio-Benzoate, Theobromin Sodio-Salicylate, Theophyllin and Euphyllin on the Coronary Flow and Cardiac Action of the Rabbit. By FRED M. SmITH and (by invitation) G. H. Miller and V. C. Graber, Iowa City, Ia.

Caffein sodio-benzoate, theobromin sodio-salicylate and theophyllin are generally believed to have a dilating action on the coronary vessels. More recently euphyllin has been said to augment the coronary flow to a far greater degree than either of the previously mentioned drugs. The investigation of the action of these drugs has resulted in varying results and in speculation on the part of the observer chiefly because of the methods employed and of the fact that the factors which influence the coronary circulation were not controlled.

The object of the present investigation was to determine the action of these drugs on the coronary vessels in concentrations that were estimated to approximate 
that in man following the administration of a therapeutic dose and in experiments in which those factors influencing the coronary flow were as nearly as possible controlled. Furthermore, the effect of euphyllin, which has far greater action on the coronary flow than either caffein sodio-benzoate, theobromin sodio-salicylate or theophyllin was compared with that of nitroglycerin which is universally employed as a vasodilator.

Experimental Coronary Embolism. By WALTER W. HAMBURger and (by invitation) W. S. Priest, JR., and Ralph B. Bettman, Chicago, Ill.

The purpose of this investigation was to attempt to reproduce experimentally, chronic interstitial myocarditis in dogs by the injection of a suspension of lycopodium spores into the coronary circulation. This was carried out in a series of twelve dogs and the immediate and remote effects of this embolic obliteration of small arterioles and capillaries were studied electrocardiographically, at varying intervals, following the injection. After death gross and microscopic studies of the heart were made.

The results of this study may be stated briefly as follows: The pre-operative injection of morphin in dogs causes typical vagus stimulation effects: ether narcosis in dogs is frequently followed by inversion of the $T$-wave and prolongation of the P-R interval: relatively widespread and sudden obliteration of arterioles and capillaries from massive lycopodium spore injections results in immediate death of the animal, the electrocardiograph showing complete $\mathrm{A}-\mathrm{V}$ dissociation, auricular flutter, or ventricular fibrillation: the injection of smaller amounts of lycopodium spores is compatible with complete recovery of the animal, the electrocardiograms in such dogs showing variously, inversion of initial ventricular complexes, right ventricular preponderance, nodal rhythm, ventricular extrasystoles, etc., with primary and secondary inversion of the T-wave. The apical areas of the hearts of these dogs show typical chronic interestitial myocarditis scarcely to be distinguished from that occurring in man as a result of atherosclerotic obliteration of the smaller coronary vessels.

Experimental Heart Disease. By George R. Herrmann, New Orleans, La.

The hearts of 200 normal dogs were divided by the author's midseptal line method and by Lewis' septum separation method. The averages for all the sectional and proportional heart weights for this control series were calculated. The most important ratios were as follows:

$$
\begin{aligned}
& \text { L/ } \mathbf{R}^{(\mathrm{H})} 1.393 ; \mathrm{L} / \mathrm{R}^{(\mathrm{L})} 1.461 ; \frac{\mathrm{Ht} . \text { Wt. }}{\text { Bd. Wt. }} 0.00798 ; \\
& \frac{\text { L.V. Wt. }}{\text { Bd. Wt. }}{ }^{(\mathrm{H})} 0.00369 ; \frac{\text { L.V. Wt. }}{\text { Bd. Wt. }}{ }^{(\mathrm{L})} 0.00306 .
\end{aligned}
$$

Experimental aortic insufficiency was produced in 75 of 150 operated dogs. Analyzed according to the time interval after operation (2 to 530 days) the maxi- 
mum increases in the above ratios, respectively $47 ; 53 ; 47 ; 72 ; 75$ per cent were found in the 70 to 110 day groups.

Comparing the heart ratios found under various experimental conditions, it was evident that of the factors that determine the degree of hypertrophy, secondary infection and the relative youth of the dog are most important. Spartein and adrenalin, as well as thyroid feeding were not conspicuously effective. Pure traumatic lesions, especially in adults, produced very moderate changes. Evidences of heart failure were noted only in dogs with endocarditis. Spontaneous endocarditis developed in 6 pups under poor hygienic conditions, in 2 parturient bitches and apparently healed in 2 bitches who had had litters of puppies and 2 who had had no puppies and in 2 male dogs.

Electrocardiograms showed definite evidence of left preponderance in only one instance, that of the dog with the maximum cardiac hypertrophy obtained.

Cinematographic Studies of Skin Capillaries in the Living Human Subject. By Alfred E. CoHN and (by invitation) J. HaMmton Crawford and H. RossenBERGER, New York, N. Y.

The method: The capillaries in the nail fold have been investigated; patients lie flat in bed with the arm stretched out horizontally at heart level. The apparatus consists of: (1) lighting system with heat filter; (2) microscope; (3) stand for holding and adjusting finger; and (4) camera. The number of exposures used was ten per second. Room temperature varied from 19 to $22^{\circ} \mathrm{C}$. The loops have been studied by projecting pictures, tracing their contour, and measuring the calibre at corresponding points (magnification 350).

Normal individuals, auricular fibrillation, mitral stenosis, chronic myocarditis and aortic incompetence were studied on consecutive days. Both limbs varied in calibre from moment to moment and from day to day in a similar manner. Changes were more marked and sudden variations occurred more frequently in cases of heart disease. Auricular fibrillation showed no dissimilarity from regular rhythm. These changes were uninfluenced by digitalization. They were unrelated to capillary contractility or individual heart beats. Some cases of aortic incompetence showed changes which suggested pulsation while others did not.

Blood flow varied in individual capillaries. In heart disease it was slower and more irregular. Digitalis improved it. Pulsation was sometimes seen in aortic incompetence. A film was demonstrated.

Vasomotor Dilatation Following Sympathectomy. By LEONARD G. RowNTREe and (by invitation) GEORGE E. BROwN, Rochester, Minn.

A group of cases in which periarterial sympathectomy and lumbar sympathectomy were performed were studied to determine the vasomotor and blood flow changes.

The first group included thrombo-angitis obliterans, Raynaud's disease, erythro- 
melalgia and endarteritis obliterans in which the Leriche operation was performed. The second group included five cases in which the lumbar sympathectomy had been carried out for the relief of spastic paraplegia. The third group included one case of malignant hypertension in which lumbar sympathectomy was carried out with the hope of decreasing systemic arterial pressure and providing an area of diminished resistance which would dilate under strain and possibly protect the cerebral vessels.

The results of the Leriche operation were practically negligible with the exception of one case of Raynaud's disease in which demonstrable vasomotor dilatation was demonstrated by the hand calorimeter and skin temperature studies. Marked vasomotor dilatation takes place in the vessels of the legs following bilateral lumbar sympathectomy as shown by the calorimetric studies of the feet, skin temperature determinations and clinical evidence. There was also complete absence of sweating.

The Effect of Various Factors on the Degree of Compensatory Hypertrophy of the Kidney after Unilateral Nephrectomy. ${ }^{1}$ By LOIS LOCKARD MACKAY and Eaton M. MacKay (by invitation) and T. Addis, San Francisco, Cal.

One kidney was removed from albino rats of known age raised under constant environmental conditions. In an equal number of animals one kidney was exposed but not removed. Each day the body-weight and the consumption of a standard casein-starch-lard-vitamine diet adequate for growth and reproduction was measured. They were kept in a constant temperature room lighted through window glass. In measuring the effect of age $\mathbf{5 0}$ rats were used at each age period and in determining the effect of food changes 100 rats were used in each experiment. The standard or experimental diet was given for 14 days before the kidney was removed or exposed and for $\mathbf{4 0}$ days after. Then the animals were killed and the kidney weights obtained. Simple hypertrophy was measured by the percentage increase of the weight of both kidneys of the exposed group on the experimental diet over the weight of the kidneys in the exposed group on the standard diet. Compensatory hypertrophy was similarly measured by comparison of the percentage increase in the remaining kidney in the nephrectomised group over half the weight of both kidneys of the exposed group. Both forms of hypertrophy are expressed as per cent body weight.

1 This work was aided by a grant from the Ella Sachs Plotz Foundation. 


\begin{tabular}{|c|c|c|}
\hline & $\begin{array}{c}\text { Simple } \\
\text { hypertrophy }\end{array}$ & $\begin{array}{c}\text { Compensa- } \\
\text { tory } \\
\text { hypertrophy }\end{array}$ \\
\hline \multicolumn{3}{|l|}{$\begin{array}{l}\text { Effect of: } \\
\text { Age-females: }\end{array}$} \\
\hline 1 month... & & 52.6 \\
\hline 3 months................... & & 36.7 \\
\hline 6 months...................... & & 32.8 \\
\hline 12 months........................ & & 32.3 \\
\hline \multicolumn{3}{|l|}{20 per cent urea diet ( 6 months - females): } \\
\hline Control $\ldots \ldots \ldots \ldots \ldots \ldots \ldots \ldots \ldots$ & 0 & 33 \\
\hline Experimental..................... & 24 & 41 \\
\hline \multicolumn{3}{|l|}{72 per cent protein diet ( 1 month-males): } \\
\hline Control $\ldots \ldots \ldots \ldots \ldots \ldots \ldots \ldots \ldots \ldots \ldots$ & $\mathbf{0}$ & 37 \\
\hline Experimental $\ldots \ldots \ldots, \ldots, \ldots, \ldots, \ldots, \ldots, \ldots, \ldots$ & 57 & 57 \\
\hline \multicolumn{3}{|l|}{ Acid diet-2 per cent $\mathrm{CaCl}_{2}(1$ month-females $):$} \\
\hline Control $\ldots \ldots \ldots, \ldots, \ldots, \ldots, \ldots \ldots \ldots$ & $\mathbf{0}$ & 53 \\
\hline Experimental........................ & 15 & 53 \\
\hline
\end{tabular}

Blood Diazo Reaction in Cases with Impaired Renal Function. By G. O. Broun and (by invitation) Margaret Riggs and O. Garcia, St. Louis, Mo.

Filtrates of blood plasma of nephritic cases after precipitation of the proteins with alcohol show a yellow color reaction when treated with Ehrlich's diazo reagent. The most marked reactions are seen in cases showing uremic symptoms.

The reaction is not given by uric acid, urea, creatine, creatinine, glucose, imidazole, phenyl alanine, guanine hydrochloride, guanidine hydrochloride, adenine hydrochloride, and adenine nucleotide. Negative results were also obtained with leucine, tyrosine, glycine, histidine, cystine, and the bile acids.

Sulfates, sulfites, thiosulfates, and thiocyanates do not give the reaction. $\mathrm{Hy}$ drogen and ammonium sulfide and organic substances containing an - $\mathrm{SH}$ group all give the reaction, e.g., ethyl mercaptan, thio-acetic acid, thiophenol, and cysteine. Carbon bisulfide, ethyl sulfide and ethyl disulfide, and diphenyl disulfide do not give the reaction.

Phenol, indole, and tryptophane also give the reaction.

While the identity of the substance responsible for the reaction in nephritic blood is not definitely established it would seem to be a type of substance whose retention has not yet been clearly shown.

The Factors Causing Acidosis in Chronic Nephritis. By Harold A. Bulger (by invitation) and JoHn P. Peters, New Haven, Conn.

In a series of cases of chronic interstitial nephritis the inorganic constituents of the serum were determined in order to give some indication as to the cause of the acidosis. The total base was determined by Cullen and Robinson's adaptation of Fiske's urine method. Bicarbonate, chloride, phosphate and protein were also 
determined by standard procedures to obtain some idea of the distribution of base among the various acids. If acidosis is defined in the terms of Van Slyke as a reduction of the bicarbonate content of the serum it appears that the acidosis of nephritis is caused by more than one factor. The molar increase of phosphate was insufficient to account for the total decrease of bicarbonate and chloride. A reduction of total base and increase of undetermined acids appeared to be the most significant factors, phosphate playing a somewhat less important rôle. Sulfate could hardly account for all the undetermined acid; presumably organic acidsalso played a part. The degree of acidosis appeared to be greatly influenced by variations of chloride. Frequently, especially with vomiting, chloride was quite low, thus allowing more base for combination with bicarbonate and resulting in only a slight reduction of $\mathrm{CO}_{2}$. With high chloride, bicarbonate was sometimes extremely low.

Acidosis Following the Feeding of $i$-Hydroxystearic Ethyl Ester. By WALTER W. PALMER and (by invitation) RANDOLPH West and ETHEL M. BENEDICT, New York, N. Y.

This investigation was undertaken to attempt to determine whether or not an even carbon fatty acid with a negative group attached to the $a, \gamma, \epsilon$ or some corresponding carbon atom would yield acetone on oxidation in the body.

i-Hydroxystearic acid was prepared by the method of Saytzeff and esterified in the usual manner. The ester was then homogenized to a cream with skimmed milk. The acetyl value of the ester was 80-85. Three normal individuals were placed on a high fat diet and when excreting about 7 grams of acetone daily about half food fat was replaced by the synthetic product. The acetone excretion dropped to 3.5 grams daily, and stool fat analysis showed that the product fed was absorbed.

It is of interest to note that though the acetone falls, organic acid output remains almost unchanged, and it seems probable that the synthetic product is at least in part oxidized and not wholly stored by the body. In one experiment, not tabulated here, in which i-oxystearic ethyl ester having an acetyl value of but 40 was fed there was no reduction in ketosis. It, therefore, appears that the non-hydroxylated fraction of the product fed is ketogenic.

The Change of the Oxygen Dissociation Curve of Blood by Carbon Monoxide and Its Significance in Carbon Monoxide Poisoning. By William C. Stadie, Philadelphia, $P a$, and (by invitation) KIRBy A. Martin, New Haven, Conn.

The effect of carbon monoxide upon the oxygen unloading function of blood is expressed in a mathematical equation theoretically deduced. Experiments in vitro on blood with varying oxygen and carbon monoxide tensions validate this equation.

By means of the equation the oxygen dissociation curve of blood, 50 per cent saturated with carbon monoxide, is plotted at a normal (7.4) and an acid (7.0) $\mathrm{pH}$ and contrasted with a normal curve.

(1) The curves predict that increase of acidity of the blood regardless of ventila- 
tory rate would increase the rate of elimination of carbon monoxide in asphyxiated animals. This was experimentally verified in asphyxiated dogs by keeping the pulmonary ventilation constant and treating with acids $\left(\mathrm{CO}_{2}\right.$ and $\left.\mathrm{HCl}\right)$. The significance of this in therapy is discussed.

(2) The profound alteration of the normal oxygen dissociation curve affords an explanation of the anoxemia of carbon monoxide poisoning. For example, in a subject whose blood is $\mathbf{5 0}$ per cent saturated with $\mathrm{CO}$, although the blood has a load of oxygen 2 to 3 times the normal physiologic requirements, its unloading tension is diminished to below $40 \mathrm{~mm}$. At this tension the oxygen is relatively unavailable for tissue metabolism and the marked symptoms of carbon monoxide anoxemia follow.

On Biliary System Function. By WIILIAM P. MurPHy (by invitation) and Reginald Fitz, Boston, Mass.

This paper reports a comparison of the Rosenthal modification of the phenoltetrachlorphthalein liver function test and the icterus index test in a series of fifty cases. The value of the icterus index as a practical and simple diagnostic test for disease of the biliary system is emphasized. A simple clinical method for determining the icterus index is described.

The Significance of Bilirubinemia as shown by the Icterus Index. By Alice R. Bennerem (by invitation) and Neliss B. Foster, New York, N. Y.

A number of estimations in diabetes mellitus show the icterus index to be high in this disease. The high index is due to a hyperbilirubinemia. Carotinemia is ruled out, as determinations were made while the patients were on a three-day milk diet. (Carotin and xanthophyll disappear from the blood after a period of twelve hours.)

A number of sugar tolerance tests were made on normal individuals to see whether high blood sugar bore any relationship to the hyperbilirubinemia. The icterus index was seen to rise with the rise in blood sugar. The same test in diabetics showed a fall in icterus index with a rise in blood sugar.

The liver was stimulated by giving food, by arousing appetite, by liver function tests and by administering a cholegogue. Under these conditions a definite relationship was shown to exist between blood sugar and bilirubinemia. In diabetes a definite but different relation was disclosed.

The liver in diabetes is disturbed in its glycogen storing capacity. Glycogen is constantly being converted into sugar. Presumably this entails a hyperactivity on the part of the liver.

The hyperbilirubinemia in diabetes seems to be another manifestation of the hyperactivity of the liver cell in this disease.

The Effects of Adrenalectomy and Adrenal Cortex Extracts in Muscular Fatigue. By J. C. Aub, and (by invitation) Walter Bauer, Boston, Mass.

The function of the adrenal cortex is unknown. There are two good ways of 
investigating it after extirpation of the adrenal tissue: (1) by finding an active cortical extract which will maintain life, or (2) analyzing physiological changes which occur. Experiments on the duration of life in cats have so far only increased our average from 32 hours in untreated animals to 55 hours in injected ones. Glucose improved marked adrenal insufficiency only temporarily.

Upon analyzing reactions of cats after adrenalectomy it seems obvious that they can make sudden movements with considerable force, but prolonged effort is impossible. We have therefore determined the amount of work individual muscles can do when left with an intact blood supply but pulling against a spring of known strength. About 17 hours after adrenalectomy such a preparation averages far less work than do normal controls. This offers a method for studying individual factors, also the effects of extracts upon this condition. Extracts which contain adrenalin increase contractions during the time of injection, but in preliminary experiments a cortical extract has given prolonged increased contractions for over twenty minutes. The correct active principle, however, should probably do this for several hours.

We are continuing these experiments.

Studies Bearing Upon the Composition of Rachitic Bone. By W. McK. MARRIOTT, B. KRAMER and J. Howland, Baltimore, Md.

From the analyses made upon the bones of children and of rats, it may be said that the calcium phosphate compound in all bones, rachitic and non-rachitic, is tricalcium phosphate. It is possible to show that the proportion of calcium phosphate to calcium carbonate is greater in the bones of the normal than in the rachitic rat. Analyses of human bones show that this is also true of children.

Precipitates made from solutions comparable in their inorganic composition to human serum show that these precipitates contain essentially the same calcium phosphate compound as bone and that the ratio of calcium phosphate to calcium carbonate, other conditions being the same, varies directly with the inorganic phosphorus content of the solution just as do the bones of rachitic and normal animals. This must not be taken to indicate a belief that calcification is a simple precipitation. There are other factors that must be taken into account.

Observations on the So-called Occult Urine Sugar with Particular Reference to the Blood Sugar Level. By L. W. Gorham, Thomas Ordway and (by invitation) Frank T. Huested, Albany, N. Y.

Specimens of blood were examined every two hours by the micro method of Randles and Grigg, and two hourly specimens of urine were examined for their sugar content by the Benedict-Folin-Hawk Method.

Curves were charted in series of cases represented in the following groups: (1) normal individuals, (2) Untreated diabetics, (3) diabetics under insulin, (4) so-called renal diabetics, (5) staphylococcus skin infections.

In the first group characteristic normal curves were determined on varying diets. Average normal curves were made which showed only slight variations in different 
normal individuals, even though the caloric intake, exercise, and mental state were inconstant. In the second group curves conforming to a single type occurred, blood and urine sugar being consistently elevated, but the urine showed much greater variations than the blood sugar. In the third group the change from the diabetic curves to normal curves as induced by Insulin was shown. In the fourth group a diagnostic relation between blood and urine curves was found in the mild and so-called renal diabetics. In the fifth group, which was studied on the supposition that recurrent furuncles might be associated with "peaks" of high blood sugar, usually missed when the specimen is taken with the patient fasting, the results were not conclusive.

The curves are of value as follows: (1) Aid in the prompt recognition of mild and so-called renal diabetics, under normal conditions of diet, as contrasted with the dextrose test meal. (2) Permit use of large doses of Insulin to obtain rapid desugarizing of patients, thus shortening hospital stay. (3) Furnish an accurate idea as to dosage, the optimum time of administration, and amount of Insulin necessary. (4) Permit the detection of cases of persistent hyperglycemia with negative urine.

Frequently Repeated Blood Sugar Curves in Non Diabetic Individuals. By STANLEY COBB and (by invitation) WM. G. LenNox, Boston, Mass.

From two to twelve blood sugar curves were done on each of 100 healthy or epileptic individuals. In a few of these individuals, the form of the successive curves varied markedly from time to time, but in most the form of successive curves was fairly uniform. In two thirds of the cases, repeated administration of glucose resulted in a progressive lowering of the sugar curve. On initial examination, about 30 per cent of the persons showed a "diabetic type" of curve. Of those who had one or more repetitions of the test, more than one half had normal curves on the second or third examination. If the administration of glucose was repeated after an hour or two, in case it was injected intravenously, the second curve was almost always slightly lower than the first. If the glucose was ingested, the second curves might be higher, lower or unchanged. On the basis of the 350 blood sugar curves made, we feel that a single observation of a high blood sugar curve, in itself, is without value. The observations suggest that in certain individuals the administration of glucose stimulates the glucose disposing mechanism of the body so that it handles subsequent doses of glucose more easily.

The Action of Adrenalin Chloride on the Circulation in Man. By H. FreLD, Jr., (by invitation) and A. V. Bock, Boston, Mass.

Despite observations in the literature that, under certain circumstances, at least, adrenalin causes vaso-dilator effects, the impression still prevails that it raises blood pressure by peripheral constriction. Our experiments on the rate of blood flow and other associated phenomena indicate that the main action of adrenalin is upon the output of the heart, which is greatly increased, and that no demonstrable peripheral constriction occurs. 
The Rate of Blood Flow as Determined by a New Method. By Francis W. PeaBody and (by invitation) Herrmann L. Blumgart and Otto C. Yens, Boston, Mass.

The method utilized is as follows. The active deposit of radium is injected at one point and its time of arrival at another point noted. In rabbits, the marginal ear vein and the foot were the two places arbitrarily chosen.

The various devices for detecting the presence of minute quantities of radium depend on the radiations continually emitted from the radium atom. These radiations can penetrate tissues and ordinary materials but can be completely stopped by sufficient thicknesses of lead. The animals are separated from the detecting device by thick blocks of lead except at one point. When the blood carrying the radium active deposit reaches that point, the radiations, instead of being stopped by the lead, will penetrate through the air and be registered by the detector.

For the purpose of detection we have utilized a needle electrode ionization chamber and also a cylindrical ionization chamber. Within the past few weeks we have had promising results using the cloud method of C. T. R. Wilson. On man, the active deposit has been injected into the vein of one arm and detected at the wrist of the other arm. At present we are making further observations on human subjects to determine more accurately the clinical feasibility of this method.

The Effect of Excluding Pancreatic Juice from the Duodenum on the Motility of the Stomach and Small Intestine. By J. H. PratT and (by invitation) L. WhITAKER, Boston, Mass.

Study was made on dogs in which the pancreas had been completely separated from the duodenum. The passage of food from the stomach through the intestines was determined by the Roentgen ray. The stomach emptied much more rapidly than in health and the passage of food through the upper portion of the small intestine was accelerated.

The Absorption of Bile Pigment from the Intestine. By M. A. BLANKEnhorn, Cleveland, Ohio.

A method is presented to study the bile contents of dogs' portal vein blood. Portal vein blood was compared with jugular vein blood as to the content of bile pigment. There is evidence to show that portal vein blood contains more urobiln than does jugular vein blood, and that absorption of urobilin takes place from the intestine.

An Hypothesis Concerning the Transportation of Water in the Body. By THOMAS E. BuckMan and (by invitation) DaN C. Darrow, Boston, Mass.

Since Starling demonstrated the magnitude of the osmotic pressure exerted by the colloids of the blood plasma, it has been generally held that the resorption of water on the venous side of the capillary meshwork is due principally to the fall in the hydrostatic pressure of the blood. 
Evidence is here presented to show that another mechanism may influence the transfusion of water between blood and tissues. This mechanism is dependent on the increase in the osmotic pressure of the blood plasma which accompanies increasing tensions of carbon dioxide to which the whole blood is exposed, a change in a general way proportional to the increase in the bicarbonate content of the plasma.

The effective pull of the blood in regions of higher carbon dioxide tension would depend, in part, on the difference between the bicarbonate content of arterial and venous plasma. This difference, in turn, would be influenced by (1) the shape of the carbon dioxide dissociation curve of the blood and (2) the differences between the tensions of carbon dioxide and oxygen in the lungs and tissues.

Some data concerning the causes of shape differences in different carbon dioxide dissociation curves are presented and also certain clinical observations which might be accounted for on the basis of the conception here set forth.

Transfusion of Lymphocytes and Their Rapid Disappearance from the Circulation of Man. By Raphael IsaAcs and George R. Minot, Boston, Mass.

Whole blood from a case of chronic lymphatic leukemia (white blood cells 89,000 per cubic millimeter; 95 per cent lymphocytes) was transfused into a patient with generalized lympho-sarcoma (white blood cells 6400 per cubic millimeter; 15.5 per cent lymphocytes; hemoglobin, 50 per cent). The lymphocytes in the peripheral circulation of the recipient increased to 42.5 per cent after the transfusion, but dropped to 25 per cent in one-half hour and reached the pretransfusion percentage within two and one-quarter hours.

Factors of Dehydration Following Pyloric Obstruction.2 By JAmes L. Gamble, M.D., and (by invitation), Munroe A. McIver, Boston, Mass.

Data obtained from rabbits consist of measurements of water, chlorides and fixed base in the gastric contents of controls and after closure of the pylorus. They show, following pyloric obstruction, a secretion of water, chlorides and fixed base into the stomach of from two to three times the estimated initial total plasma content.

A chief point of these findings is the large loss of fixed base. From the point of view of dehydration, the loss of base is the significant factor since it represents an absolute depletion of the body's content of dissolved electrolytes, whereas loss of chloride ion is replaced by increase of bicarbonate ion.

Haden and Orr regard the action of sodium chloride as protective against a toxic substance rather than simply reparative of dehydration. They state that the chloride lowering in the plasma is only in part explained by loss in vomited secretions. They find that it occurs when there is little vomiting and in rabbits which

${ }^{2}$ A complete report of this work appears in The Journal of Cinncal InvestuGATION, 1925, i, 531. 
cannot vomit. They believe that chloride leaves the plasma in offensive quest of a toxic substance.

Our rabbits did not vomit. They, however, lost into their stomachs several times the total plasma capacity for chloride. That any chloride at all is found in the plasma proves a movement in the direction opposite from that surmised by Haden and Orr.

The Effect of Iodine by Mouth on the Reaction to Intravenous Injections of Thyroxin.

By Cyrus C. Sturgis and (by invitation) Salvador Zubiran, Guy W. Wells and THEODORE BADGer, Boston, Mass.

The recent observation that the oral administration of iodine results in a striking reduction in the basal metabolism of patients with exophthalmic goiter adds new information to our knowledge which may have important bearing on the physiology and physiological pathology of the thyroid. It is not clear, however, whether the principal mode of action of iodine in producing this remission is by causing some anatomical alteration in the thyroid gland or by exerting some effect on the product secreted by the gland. In an endeavor to gather further information on this subject, rabbits were injected with small doses $(1 \mathrm{mgm}$. on 3 successive days) of thyroxin, and their response determined by recording their pulse rate, body-weight, and oxygen consumption daily for a long period. The same animals were then given Lugol's solution orally for a variable period and again received similar injections of thyroxin. There was no evidence that the iodine had any effect on the action of thyroxin as the animals responded in the typical manner. If exophthalmic goiter is due to pure excess of the thyroid secretion, which some hold, then the action of iodine in producing a remission is not due to its effect on the circulating thyroxin.

The Static and Kinetic Representations of the Efferent System in the Psychic Sphere. By J. Ramsay HunT, New York, N. Y.

In previous communications the dual nature of the efferent nervous system has been presented and its bearing on the interpretation of motor disorders. According to this conception, movement is subserved by a kinetic, and posture by a static mechanism, which present a parallelism of function and structure at all physiological levels of the efferent nervous system, vegetative and cerebrospinal.

This paper considers the kinetic and static representations of the efferent system in the purely psychic sphere. Evidence is presented in favor of the view that thinking is a kinetic representation; while decision, conclusion and belief are representations of static function. Therefore movement and posture are represented in mental processes by thinking and belief respectively. The relation of thinking and belief to certain morbid mental processes is considered. 


\section{The Resistance of Immature Erythrocytes to Heat. ${ }^{3}$ By RAPHAEL IsAacs and}

George R. MnNot, Boston, Mass.

The red blood corpuscles of normal human blood, when heated to $55^{\circ} \mathrm{C}$. for one-half hour, undergo a profound and characteristic modification, with the production of fragmented forms, "shadows," microcytes, poikilocytes and a uniform distribution of the hemoglobin throughout the cell. No noteworthy visible changes take place at $45^{\circ} \mathrm{C}$. to $50^{\circ} \mathrm{C}$. but at $65^{\circ} \mathrm{C}$. complete destruction occurs. Immature erythrocytes, reticulocytes, polychromatophilic cells and granule red cells, of both normal and pathological blood are much more resistant when heated to $55^{\circ} \mathrm{C}$. than mature erythrocytes, with the apparent exception of reticulated megalocytes. The difference between the effect of heat on the red cells of normal and pathological blood is not qualitative; it is quantitative, proportional to the number of immature cells.

There are two kinds of red cells which show no histological evidences of immaturity, and are classed as mature corpuscles. One kind resists the action of heating to $55^{\circ} \mathrm{C}$. while the other is broken up and altered. The former, represent a majority of the red blood corpuscles in chronic hemolytic jaundice so that the bulk of the red cells of this condition remain conspicuously intact after heating. They are probably the younger of the mature cells.

\section{A Report of Fifty Patients suffering from Graves' Disease and under Observation} for Four Years. By Leo Kessed and (by invitation) H. T. HyMaN, New York, N. Y.

A report of the further progress of fifty patients who suffered from Graves' Disease and who are now at the end of the fourth year of observation, who have received no specific therapy, whose progress has been estimated by subjective symptoms, by objective methods particularly pulse rate and basal metabolism, exophthalmometer readings and weight, and by a standard as to their economic and social restitution. This report is to serve as a control to a series later to be reported, in which the same régime was followed except that a sub-total thyroidectomy was done on series I and in series II Lugol's solution was administered before thyroidectomy was done.

It is hoped that at the completion of the observations of these series a definite therapeutic policy can be established for the management of patients suffering from Graves' disease.

Studies of the Vascular Features of Polycythemia Vera. By George E. Brown (by invitation) and Herbert Z. GrFrin, Rochester, Minn.

The basis of the paper is the study of fourteen cases. An increased viscosity was present in all. The highest reading was 1:11.2 (normal 1:4.5). With a

A complete report of this work appeared in The Journal of Clinical INvestigation, 1925, i, 425. 
normal red cell count the viscosity was frequently increased. The total circulating blood volume was increased in all cases, the average being $10,300 \mathrm{cc}$. or $166 \mathrm{cc}$. for each kilogram. Cell hematocrit values varied from 58 to 70 and averaged 62 . In twelve cases in the nail fold the venous limb of capillaries and the collecting venules were markedly engorged, and in four cases the arterial limb was engorged. All available capillaries were utilized. The capillaries reached their normal size when the total circulating blood volume had decreased to $106 \mathrm{cc}$. for each kilogram. Engorgement of the retinal veins disappeared at the same volume. Cardiac enlargement and cardiac lesions were noticeably absent. The liver was only slightly enlarged. The size of the spleen bore a direct relationship to the degree of total circulating blood volume. The engorgement of the vessels and the increase in viscosity had no effect on the elevation of arterial tension. The venous pressures were slightly higher than normal; the capillary pressures were normal. Renal function was only slightly impaired. Heat production in the foot showed abnormally marked fluctuations. In polycythemia, the capillaries and venules have a storage function; this in association with retardation of flow affects fundamentally the mechanism of heat dissipation.

Energy Expenditure during Mechanical Work in Obese, Normal, and Thin People.

By Solomon Strouse and (by invitation) C. C. WANg and Zelma Owen, Chicago, Ill.

Growing out of the investigations of the energy metabolism of obesity a comparison of the energy expended for mechanical work among obese, normal, and thin subjects was undertaken. Tissot respiration apparatus was employed as in our previous work. The energy expended for mechanical work was measured by a bicycle ergometer.

Thirteen obese, ten normal, and six thin people served as subjects. Normals were those whose weights varied between plus ten and minus ten per cent from their standard weights.

Results are expressed in terms of mechanical efficiency. Figures show that the obese spend more energy on a given piece of work than the normal, and the thin people are more efficient than either of the other groups. The respiratory quotients are invariably increased during exercise and the increase is directly proportional to the mechanical efficiency of the three groups.

The Reactivation of Inactivated Insulin in Vitro and in Vivo. By ALBERT A. EPsteIn, New York, N. Y.

Previous studies have established that trypsin can inactivate insulin in vitro and in vivo, and that this function in vivo may play an important rôle in the causation of diabetes.

Insulin may be dissociated from trypsin in vitro by shifting the hydrogen ion concentration of the substances in solution to the acid side of 4.6. It became necessary to ascertain whether dissociation could be effected in vivo. Obviously 
the hydrogen ion concentration of the body fluids could not be altered to the point necessary for the dissociation of insulin. Hence other means were tried and these results obtained:

1. Upon addition, in vitro, to inactivated insulin (trypsulin) of such substances as pepsin, safranin, and cryogenin (m-benzaminosemicarbazide) liberation of insulin takes place, as evidenced by results obtained from injection of these mixtures into suitable test animals.

2. These agents (pepsin, safranin and cryogenin) can dissociate insulin from trypsin in vivo. Injection of suitable amounts of these substances (subcutaneously or intravenously) just prior to parenteral administration of inactivated insulin causes its liberation and the production of its physiological effects.

Therefore if diabetes is the result of insulin deficiency caused by inactivation of insulin by trypsin, then the possibility of reactivating insulin in vivo may be of practical therapeutic importance.

Absorption Curve of Spinal Fluid. By A. T. SHOHI and (by invitation), S. KARELITs, New Haven, Conn.

A study is being made of the acid-base equilibrium of spinal fluid, to apply to physiological and clinical problems. The absorption curve has been studied by saturation with the Van Slyke et al. technique by use of a new type of tonometer which permits both saturation and colorimetric pH determination and sampling for $\mathrm{CO}_{2}$ content without transfer or exposure to air. By this method, values for $\mathrm{pK}_{1}$ are obtained which permit calculation of the tension of $\mathrm{CO}_{2}$ in spinal fluid without requiring absorption curves.

The Influence of Temperature on Acid Base Equilibrium in the Blood. By J. H. Austin and (by invitation) G. E. Cullen and H. W. RoBinson, Philadelphia, $\mathrm{Pa}$.

In the study of blood acid base equilibrium under conditions of varying temperature there are four variables of which the temperature coefficient must be taken into account.

1. The solubility of $\mathrm{CO}_{2}$.

2. The value of $\mathrm{pK}^{\prime}$ of the Henderson-Hasselbalch equation. Cullen, Keeler and Robinson found

$$
\frac{\Delta \mathrm{pK}^{\prime}}{\Delta \mathrm{t}^{\circ} \mathrm{C}}=-0.005
$$

3. Change in the location of the $\mathrm{CO}_{2}$ absorption curve. This is best stated as the change in $\mathrm{pH}$ with temperature at constant $\left[\mathrm{BHCO}_{3}\right]$. Stadie and Martin found for whole human blood:

$$
\frac{\Delta \mathrm{pH}}{\Delta \mathrm{t}^{\circ} \mathrm{C}_{\left[\mathrm{BHCO}_{3}\right] \text { constant }}}=-0.022
$$


We find this value to be $-0.02 \pm 0.003$ for whole blood, true serum and separated serum of both dog and sheep.

4. The change in the neutral point, i.e., $1 / 2 \mathrm{pK}$ water. Between $10^{\circ}$ and $45^{\circ} \mathrm{C}$. this is about:

$$
\frac{\Delta\left(\frac{1}{2} \mathrm{pK} \text { water }\right)}{\Delta \mathrm{t}^{\circ} \mathrm{C}}=-0.014
$$

The Coating of Bacteria by Agglutinin in Specific Bacterial Agglutination. By Gerald S. Shibley (by invitation) and A. R. Dochez, New York, N. Y.

The specific charge reducing effect of agglutinating sera observed in work with paratyphoid bacilli and pneumococci has been determined for ten additional organisms. Where the cataphoretic charge is high without serum, it is specifically reduced; where low, it is raised. In all cases, no matter what the initial charge (range, 40 to $5 \mathrm{mv}$ ), high concentrations of sera bring the charge to a common potential level ( 8 to $14 \mathrm{mv}$ ). Loeb has shown that protein coated collodion particles take the cataphoretic charge of these proteins. Sensitized pneumococci compared with pneumococcus antiserum euglobulin particles (agglutinin) show identical charges. Probably, therefore, the first step in specific agglutination is selective coating of bacteria by specific agglutinins, and charge changes observed are the result of this. Loeb has shown that collodion particles coated with crystallin egg albumin behave, as to stability in suspension, as if the albumin film were denatured. Bacteria treated with agglutinating sera (coated) act also like denatured proteins. It is probable that the specific globulins of agglutinating sera that coat bacteria are similarly denatured and that the resulting flocculation is due to this "denaturation." Preliminary experiments with globulins and collodion particles bear out this explanation of the mechanism of specific bacterial agglutination.

Interruption of Complete Heart Block by Sequential Beats in Early Diastole Exemplifying Recovery Phase of Cardiac Muscle. By Chardes C. Wolferth, Philadelphia, Pa.

A patient in whom prolonged conduction periods were observed for two years finally developed complete dissociation, although sequential type of beating could be temporarily restored by atropin. Several times, tracings were obtained showing interruption of complete dissociation by response to auricular beats falling within a certain range of early diastole. This temporary recovery of ability to transmit impulses has been reported by Lewis and Master who attribute it to "supernormal recovery phase" described by Adrian and Lucas. The number of clinical cases now described (three) undoubtedly demonstrates a tendency during heart block toward a recovery phase of junctional tissues in early diastole. That this recovery phase is of the nature of the response described by Adrian and Lucas involves assumptions that can not be established at present. 
The Influence of Various Factors upon the Length of Systole in Man as Measured by the Electrocardiogram. By Howard B. SpRague (by invitation) and PaUL D. WHrTe, Boston, Mass.

Our investigations have verified the well known fact that the most obvious influences determining the length of systole are the factors which control the rate of the heart. These are probably various influences concerned in the nervous mechanism of the heart beat. The duration of systole in a normal control series of adult males and females and children has varied from 0.4404 second at a pulse rate of 53 , to 0.2637 second at a rate of 120 . In addition to this normal relationship between heart rate and systolic length the influence of other factors, such as cardiac failure, blood pressure, heart size, drug therapy, exercise, and hyperthyroidism are being !studied.

A Preliminary Note on the Blood and Cerebrospinal Fluid Chlorides in Chronic Nephritis and Uremia. By JoHn B. Youmans (by invitation) and FranK N. Wrison, Ann Arbor, Mich.

Studies of the influence of various ions in convulsive states, as tetany, and the investigations by Weed and Wegeforth and by Collip on the effect of intradural injections of electrolytes, suggest that an increase in cerebrospinal fluid chlorides may play a rôle in the production of convulsive uremia.

To investigate this possibility the concentration of chlorides in the blood and cerebrospinal fluid of patients with chronic nephritis, with and without uremia, was determined.

Twenty-one patients were studied. Seven developed convulsive uremia. Of the seven, four had 800 to $840 \mathrm{mgm}$. of chlorides per $100 \mathrm{cc}$. of cerebrospinal fluid, and one $770 \mathrm{mgm}$. Two had normal amounts, but agonal changes may have influenced the results. Fourteen patients without uremia gave normal findings, except one who had $792 \mathrm{mgs}$.

Simultaneous blood chloride determinations showed increased concentrations in patients with increased chlorides in the cerebrospinal fluid; but increased blood chloride concentrations occurred independently of increased cerebrospinal fluid chlorides or uremia. No constant relation was established between increased spinal fluid chlorides and such factors as blood non-protein nitrogen, blood pressure, edema, etc.

Eleven patients without nephritis (normal, syphilis, cardiac failure, etc.) gave low or normal figures for the chlorides of the cerebrospinal fluid.

Intracardiac Fistulae. By C. S. Beck (by invitation), E. C. Cutler and (by invitation) Emre Holman, Cleveland, Ohio.

Experiments were performed in which fistulae between the heart chambers were established in an attempt to study the circulation under the conditions imposed in certain forms of congenital heart disease. It appeared to us that the dilatation of the heart chambers and the muscular hypertrophy found repeatedly 
at autopsy in cases of patent interauricular and interventricular septa were unexplained by previous conceptions. It was our feeling that the interpretation of peripheral fistulae, as demonstrated experimentally and clinically by one of us might well be applicable to these congenital conditions; i.e., the changes might be due to increased blood flow through that portion of the system into which the larger stream of blood is diverted by the defect.

Interventricular fistulae were established by the use of knives and the modified cardiovalvulotome, used by us in the establishment of valvular insufficiencies. Under these conditions studies of blood pressure, blood volume, changes in the heart size as demonstrated by the $\mathrm{X}$-ray, and subsequent examinations were conducted. These studies demonstrated that the capacity of the chambers of the heart and the alterations in their musculature seem to be determined by the minute volume flow through them.

Metabolism Studies in Exophthalmic Goiter Complicated by Diabetes. By WALTER M. BоothBy and RUSSELL M. WILDER, Rochester, Minn.

Approximately complete metabolism data on two patients having exophthalmic goiter complicated by diabetes were presented. These studies, supported by several other cases less completely investigated, showed that the exophthalmic goiter syndrome materially reduces the ability of a diabetic patient to utilize carbohydrate, decreases the efficiency of a unit of insulin and increases the danger of a sudden onset of diabetic coma. The marked improvement in the ability of the patient to utilize carbohydrate, as the exophthalmic goiter syndrome is controlled by iodin (Lugol's solution), was clearly brought out. The clinical importance of recognizing the exophthalmic goiter complication in cases of diabetes was emphasized and it was pointed out that this complication should be suspected as the possible significant factor in those diabetic patients who are not readily controlled by ordinary measures.

Thymus Enlargement. By H. Grax, Santa Barbara, Cal.

In any child whose growth is retarded, thymus enlargement merits a thought. Three suggestive cases follow.

A boy aged six years was coughing. X-ray showed a broad shadow in the second interspace. Diagnosis of tuberculous nodes was rejected in favor of the thymus. After the photograph alone, the cough improved and was completely relieved after three radiations. Re-ray showed diminution of the shadow. During the ensuing year growth was rapid: in height 45 per cent and in weight 131 per cent more than the average yearly increase for his age.

A second boy, aged nine years, was backward compared with his brother a year younger, height was 10 per cent sub-normal, teeth were notched and occlusion poor, right testicle intra-abdominal. Thymus enlargement was suspected, confirmed by x-ray. Radiated six times and being watched for acceleration in development. 
A girl aged seven years, routinely rayed prior to tonsillectomy, showed thymus hypertrophy. After three radiations, weight increased about 7 pounds within a month.

The Mechanism of Death from Quinidine and Methods of Resuscitation. An Experimental Study. ${ }^{4}$ By Burgess Gordon and Marcel MatTon (by invitation) and S. A. LEVINE, Boston, Mass.

Unexpected and unexplained deaths have occasionally occurred during the clinical administration of quinidine in treatment of certain heart conditions. One fatality we observed showed a peculiar difficulty in breathing and presented a picture of a more general toxic state than is ordinarily seen with heart failure. Death was gradual and autopsy showed no evidence of emboli.

With the above experience in mind, the method of death in quinidine was studied in cats. It was found that although definite heart intoxication took place, a most important factor that has been hitherto neglected was also involved, i.e., the respiratory mechanism. It was a constant finding that the respiration failed before death and that this failure of respiration could not be entirely accounted for by a lowered state of the circulation. It was also found that when respiration had ceased, following such doses of quinidine as many previous experiments had indicated to be fatal, if artificial respiration were instituted, the animal could be saved and complete recovery take place. The administration of caffene had a similar action in resuscitation, but was not as dependable. The best and most speedy results were obtained when both artificial respiration and caffene were employed.

The Storage of Inorganic and Food Iron in the Liver, Spleen and Bone Marrow. By Charles Spencer Williamson, Chicago, Ill.

The question as to the storage of iron has been one that has been answered both pro and con. The question is intimately connected with the formation of hemoglobin. In a series of experiments, which have been carried on for about two years, the results of which are being reported at another meeting, the writer has shown that inorganic, medicinal iron may be stored up in large quantities in these organs.

The present research has been undertaken to see whether true organic iron, that is food iron, is stored up in the same way, and if so, whether such storage adds to the available reserve supply of hemoglobin. Along with this, the behavior of the blood hemoglobin has been studied with methods of precision, spectrophotometrically.

This question is of considerable importance in that it points out a possibility of

4A complete report of this work appears in The Journal of Clinical INvESTIGATION, 1925, i, 497. 
increasing the available reserve supply of hemoglobin, and especially so since there is no considerable reserve supply of iron in the body, almost 90 per cent of it being found in the blood.

Mitral Stenosis after the Fifth Decade of Life. By ERnst P. BOAS and (by invitation) David Perla, New York, N. Y.

Mitral stenosis is not uncommon in the sixth and seventh decades of life. Fortysix of 183 consecutive cases of mitral stenosis studied at Montefiore Hospital were over 50 years of age. In most instances it is the end result of a rheumatic infection in childhood or early adult life. The mitral narrowing is not extreme and the lesion is nonprogressive, or very slowly progressive, so that significant symptoms do not develop for many years. Women with such a condition may apparently undergo many pregnancies without distress and without injury to the heart.

In a certain number of cases of mitral stenosis in elderly persons, the valvular lesion cannot be interpreted as the end result of an ancient endocarditis but must be regarded as a primary atherosclerosis of the mitral valve and particularly auriculoventricular ring. The characteristic pathological finding is a widespread calcification of the mitral ring, encroaching on the lumen of the auriculoventricular opening with thickening and at times calcification and fusion of the valve cusps.

Cardiographic Differentiation of a Sub-group of Intra-ventricular Block with Observations on the Prognosis. By B. S. OpPENHEIMER, M. A. RothSCHIL, and (by invitation) HUBERT MANN, New York, N. Y.

In a series of ten patients a type of electrocardiogram was observed with such characteristics that theoretical considerations led us to believe the prognosis would be less serious than in the usual type of arborization block. The electrocardiograms of this group show marked widening and notching of the Q.R.S. complex, but they differ from the usual arborization block in that the changes are confined almost entirely to the terminal portion of the second limb of the $R$ wave. The Q.R.S. portion of the electrocardiograms bear a striking resemblance to one another. The voltage, $T$ wave, and P-R interval present no noteworthy abnormalities. The teleor-entgenograms show moderate widening of the arotic arch.

In a previous series of intraventricular block, the mortality was 52.1 per cent and the average duration of life after the initial electrocardiogram was only 8 months. In contrast to this poor prognosis, these ten patients with a solitary exception, showed no downward progress either clinically or electrocardiographically. The patients have been followed for from 1 to 9 years, an average of $3 \frac{1}{2}$ years. The most recent case, and the only syphilitic of the series, died of a lobar pneumonia. The remaining nine are ambulatory and follow their customary occupations. 